\title{
Comparative virulence of seasonal viruses responsible for lower respiratory tract infections: a southern European multi-centre cohort study of hospital admissions
}

\author{
André Almeida ${ }^{1,2}$ (1) Matteo Boattini ${ }^{3} \cdot$ Eirini Christaki ${ }^{4,5} \cdot$ Torcato Moreira Marques $^{1} \cdot$ Inês Moreira $^{6}$. \\ Lourenço Cruz ${ }^{1}$. Valentina Tosatto ${ }^{1,2}$. Diogo Antão ${ }^{1}$. Gabriele Bianco ${ }^{3} \cdot$ Marco lannaccone $^{3}$. Cristina Costa ${ }^{3}$. \\ Georgios Tsiolakkis ${ }^{5} \cdot$ Elina Khattab $^{4} \cdot$ Diamanto Kasapi $^{4} \cdot$ Ana Teresa Ferreira $^{1} \cdot$ Rossana Cavallo $^{3} \cdot$ Rita Corte-Real $^{7}$
}

Received: 21 August 2020 / Accepted: 14 December 2020 / Published online: 3 January 2021

(c) Springer-Verlag GmbH, DE part of Springer Nature 2021

\begin{abstract}
Objectives Our study aimed to compare the clinical severity of lower respiratory tract infections (LRTI's) caused by Influenza and Respiratory Syncytial Virus (RSV).

Methods We conducted a retrospective cohort study of LRTI admissions with positive PCR results for Influenza or RSV from 2017 to 2019 in three teaching hospitals in southern Europe. Data on clinical characteristics, viral agents and disease outcome were collected. Nosocomial infection was excluded. Main outcomes were invasive mechanical ventilation and inhospital death.

Results A total of 984 patients were included. Median age was 75 years. Influenza A was the most frequently identified virus (56.5\%), of which $27.1 \%$ were subtype $\mathrm{H} 1 \mathrm{~N} 1$ and $53.0 \% \mathrm{H} 3 \mathrm{~N} 2$. Influenza B was isolated in $22.3 \%$ and RSV in $21.0 \%$. There were $10.5 \%$ of patients who died during admission and $8.3 \%$ submitted to IMV. Influenza A H1N1 was associated with lower age and less co-morbidity, while the opposite was observed for RSV. Influenza A H1N1 was independently associated with both higher risk of death (adjusted odds ratio 2.0 [1.2-3.4] $p=0.008$ ) and IMV (adjusted odds ratio 5.1 [3.0-8.5] $p<0.001$ ). Conclusion Influenza A H1N1 was an independent predictor of mortality and IMV. These findings may have implications on hospital resource planning and vaccination policies.
\end{abstract}

Keywords Influenza $\cdot$ Human $\cdot$ Respiratory syncytial virus $\cdot$ Virulence

\section{Introduction}

Lower respiratory tract infections (LRTI's) are estimated to rank among the five leading causes of death and years of life lost worldwide [1]. Among its main etiological agents, Influenza is recognized as the most important virus, being responsible for a significant mortality burden $(4 \cdot 0-8 \cdot 8$ yearly

André Almeida

andre.almeida@chlc.min-saude.pt

1 Department of Internal Medicine 4, Central Lisbon University Hospital Centre, Rua de Santa Marta 50, 1169-024 Lisbon, Portugal

2 NOVA Medical School, Campo Mártires da Pátria 130, 1169-056 Lisbon, Portugal

3 Microbiology and Virology Unit, University Hospital Città Della Salute E Della Scienza Di Torino, Corso Bramante 88, 10126 Turin, Italy associated respiratory deaths per 100000 individuals) [2], which peaks seasonally over the winter months in the northern hemisphere in association with lower temperatures [3]. There is considerable inter-seasonal variation regarding Influenza-associated hospitalizations and mortality, owing to differing viral transmissibility and virulence, as well as vaccine coverage and effectiveness [4-6].

4 Medical School, University of Cyprus, Palaios dromos Lefkosias Lemesou 215/6, 2029 Aglantzia, Nicosia, Cyprus

5 Department of Medicine, Nicosia General Hospital, Nicosia, Limassol Old Road 215, 2029 Strovolos, Nicosia, Cyprus

6 Department of Pneumology, Central Lisbon University Hospital Centre, Rua de Santa Marta 50, 1169-024 Lisbon, Portugal

7 Department of Clinical Pathology, Central Lisbon University Hospital Centre, Rua José António Serrano, 1150-199 Lisbon, Portugal 
Respiratory Syncytial Virus (RSV) is increasingly recognized as an important cause of LRTI's [7] and LRTI-related hospitalizations among older adults [8], with considerable seasonal co-circulation with Influenza [9]. Yet, evidence on its burden on the adult population is scarce and at times conflicting.

Severe acute respiratory syndrome coronavirus 2 (SARSCOV-2) is a novel virus responsible for an unfolding pandemic of primarily respiratory disease which has been described for the first time in December 2019 in Wuhan, China [10]. So far, despite the report of one co-infection case in China [11], there has not been considerable report of incidence overlap between SARS-COV-2 and Influenza or RSV in Europe, as the former gained epidemiological momentum at a time when the Influenza season in Europe is usually fading. Nevertheless, there could be co-circulation of these different viral agents in the future, which could present an immense challenge to different components of healthcare.

Knowledge on microorganism virulence is of paramount importance for clinicians when addressing LRTI's to better estimate disease course, thus improving clinical judgement and decisions. In this work, we aimed to compare the virulence of common seasonal respiratory viruses implicated in LRTI-related hospital admissions before the COVID-19 pandemic, by means of studying the association between microorganisms and clinical outcomes.

\section{Methods}

\section{Study setting}

We conducted a retrospective multi-centric cohort study encompassing two winter seasons in southern Europe. Recruitment periods were October 2017-April 2018 and October 2018-April 2019. Study subjects were recruited based on positive polymerase chain reaction (PCR) results for Influenza or RSV on oral/nasopharyngeal samples, obtained over the aforementioned periods in three teaching hospitals in Cyprus (Nicosia), Italy (Turin) and Portugal (Lisbon).

\section{Study subjects and variables}

We included patients $\geq 18$ years old who were admitted to a hospital ward and/or intensive care unit for acute illness. For patients with more than one positive PCR in one seasonal period, the first episode was considered for study purposes. We excluded samples obtained from health-care workers in the setting of occupational health screening and those from patients discharged to an outpatient setting within $24 \mathrm{~h}$. Pregnant patients were excluded as were those deemed to have nosocomial infection, which was considered when both LRTI-related symptom onset and PCR sampling took place $>72 \mathrm{~h}$ after admission. Co-infections with more than one virus or more than one Influenza type/subtype were also excluded.

We collected data on demographics, co-morbidities, laboratory molecular identification, radiological presentation, clinical course until discharge or death and outcome of admission. Co-morbidities were assessed on the basis of baseline clinical history depictions and/or assessments made during admission which determined their presence. Patient smoking status was considered when current at the time of admission, malignancy when active, heart failure if class II NYHA disease or worse, chronic kidney disease stage 3A disease or worse. Laboratory identification, typing and subtyping results for Influenza (A H1N1, A H3N2 and B) and RSV were made using multiplex PCR on oral and nasopharyngeal swab specimens using Cepheid's GeneXpert Flu/RSV ® (Sunnyvale, CA, USA), Biomerieux's Filmarray respiratory panel @ (Marcy-l'Étoile, France) and Seegene's Allplex respiratory panel ® (Seoul, Republic of Korea).

Primary outcomes were in-hospital death and invasive mechanical ventilation (IMV), secondary outcomes were non-invasive ventilation (NIV) and length of stay. Pneumonia was considered when a radiological infiltrate was present on the chest X-ray and/or CT scan performed on presentation. Use of CPAP was not considered as NIV.

\section{Statistical analysis}

Normality of distribution was assessed using Shapiro-Wilk test. Associations between baseline clinical variables and microorganism infection were assessed using $\mathrm{Chi}^{2}$ for categorical predictors and Kruskal-Wallis equality of populations rank test for continuous predictors. Univariate logistic regression was performed to test associations between virus type or clinical predictors and categorical outcomes. Clinical predictor variables with $p$ values $\leq 0.1$ on univariate analysis were carried forward to the multivariate model to control for confounding. One-way analysis of variance (ANOVA) with Bonferroni correction was performed to test linear outcomes (length of stay) according to microorganism. $P$ values $\leq 0.05$ and $95 \%$ confidence intervals were considered for purposes of clinical significance. Multivariate regression results are presented as adjusted odds ratio (aOR). Statistical analysis was conducted using software package STATA 13.1 (College Station, Texas, USA) and R (R: A language and environment for statistical computing. R Foundation for Statistical Computing, Vienna, Austria).

\section{Ethics}

Patient data were anonymized and subject identifiers encrypted in the final datasets. Only the principal 
investigator in each one of the three study sites had access to the key decoding the encrypted subject numbers to local hospital file numbers. The study was conducted in accordance with the principles stated in the Declaration of Helsinki and was approved by the institutional review board of the coordinating centre (Centro Hospitalar de Lisboa Central, number 762_2019) and by the Cyprus National Bioethics Commitee (EEBK EP 2019.01.137).

\section{Results}

A total of 984 patients met the study eligibility criteria, of which $45.4 \%(n=447)$ were recruited in the 2017-2018 season and 54.6\% $(n=537)$ in the 2018-2019 season (Fig. 1). The sample obtained allowed for statistical power $>70 \%$ on ascertaining a $10 \%$ incidence difference of main outcomes between viral agents (both between Influenza types and subtypes and between these and RSV).

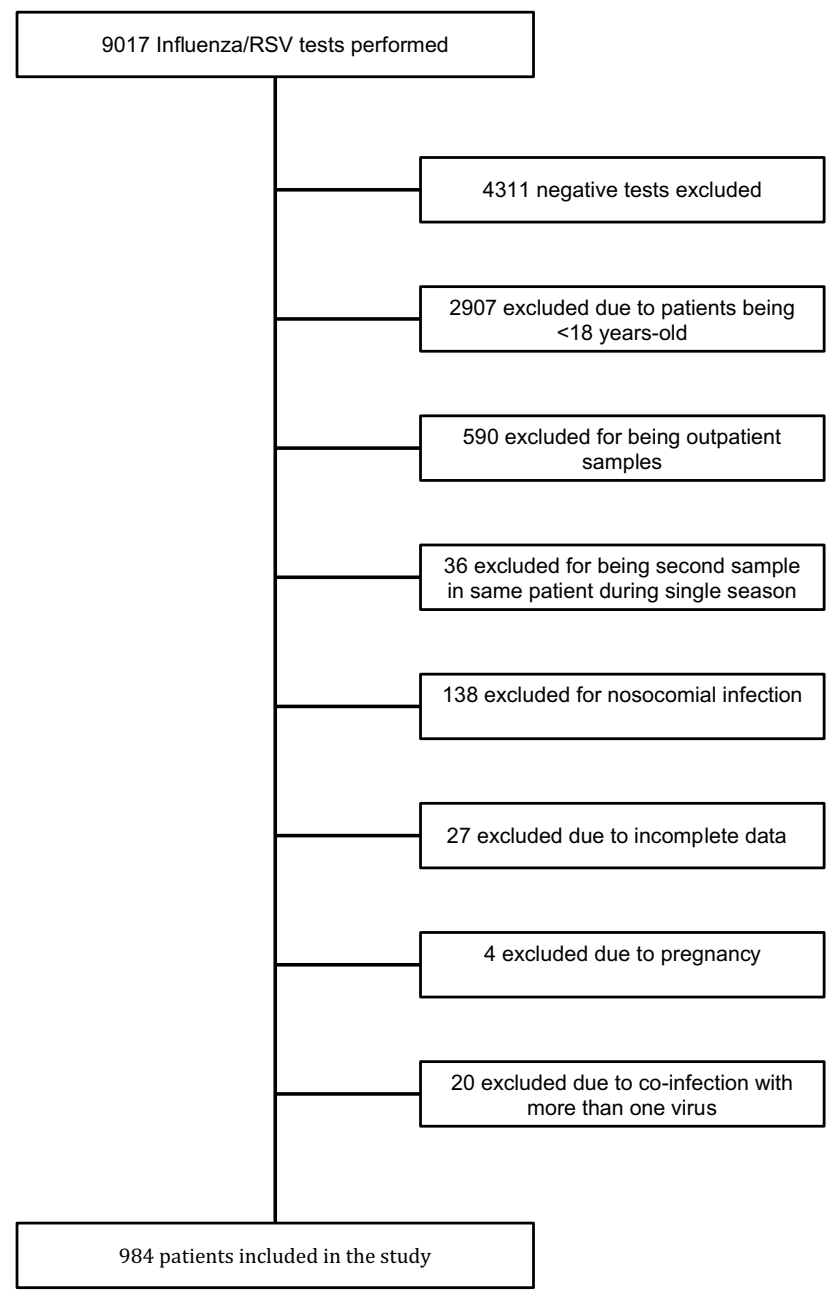

Fig. 1 Study participant flowchart
Patient demographic and clinical characteristics are presented in Table 1. At least one co-morbidity was present in $80.2 \%(n=789)$ of all patients.

Influenza A was the most frequently identified virus, accounting for $56.5 \%(n=557)$ of all infections (Fig. 2). Among these, H1N1 was isolated in $27.1 \%(n=151)$ and H3N2 in 53.0\% $(n=295)$. No subtyping data was available for 111 isolates. Influenza B was isolated in 22.3\% $(n=220)$ and RSV in 21.0\% $(n=207)$. During the 2017-2018 season, the most frequently isolated virus was Influenza $B$, whereas during 2018-2019, it was Influenza A. Regarding the 20 cases of co-infections, we found, half of these consisted of Influenza A plus RSV $(n=10)$, the other half split equally split between Influenza A plus Influenza B $(n=5)$ and Influenza B plus RSV. There were no cases of co-infection by different Influenza A subtypes.

Prevalence of comorbidities according to isolated virus is presented in Table 2.

A radiological image compatible with pneumonia was present in $36.2 \%(n=356)$ of all admissions. Influenza A H1N1 was associated with higher likelihood of pneumonia on presentation (OR $2.2[1.6-3.1] p<0.001)$, while $\mathrm{H} 3 \mathrm{~N} 2$ was associated with lower likelihood (OR 0.6 [0.4-0.8] $p<0.001)$. No other viral agents were significantly associated with pneumonia. Oseltamivir was administered to 83.4\% ( $n=648)$ of LRTI's caused by an Influenza virus.

A total of $10.5 \%(n=103)$ of patients died during the course of admission, $8.3 \%(n=82)$ were submitted to IMV, $13.6 \%(n=134)$ to NIV. Median length of stay was 9 days (interquartile range [6-15]).

Table 1 Patient characteristics and co-morbidities

\begin{tabular}{lll}
\hline & $\%$ & $n / \mathrm{IQR}$ \\
\hline Male & $48.2 \%$ & 475 \\
Age (median) & 75 & {$[62-84]$} \\
Smoking & $15.7 \%$ & 154 \\
Diabetes & $25.5 \%$ & 251 \\
CKD & $15.9 \%$ & 157 \\
HF & $30.2 \%$ & 298 \\
CLD & $3.5 \%$ & 34 \\
COPD/asthma & $29.0 \%$ & 286 \\
ILD & $1.5 \%$ & 15 \\
OSA/OHS & $5.0 \%$ & 49 \\
Solid organ transplant & $2.2 \%$ & 22 \\
Solid neoplasm & $6.0 \%$ & 59 \\
Hematological neoplasm & $11.7 \%$ & 115 \\
HIV & $2.1 \%$ & 21 \\
\hline
\end{tabular}

$I Q R$ interquartile range (presented for age), $C K D$ chronic kidney disease, $H F$ heart failure, $C L D$ chronic liver disease, $C O P D$ chronic obstrutctive lung disease, ILD interstitial lung disease, OSA/OHS obstructive sleep apnea/obesity-hypoventilation syndrome, $H I V$ human immunodeficiency virus (chronic infection) 


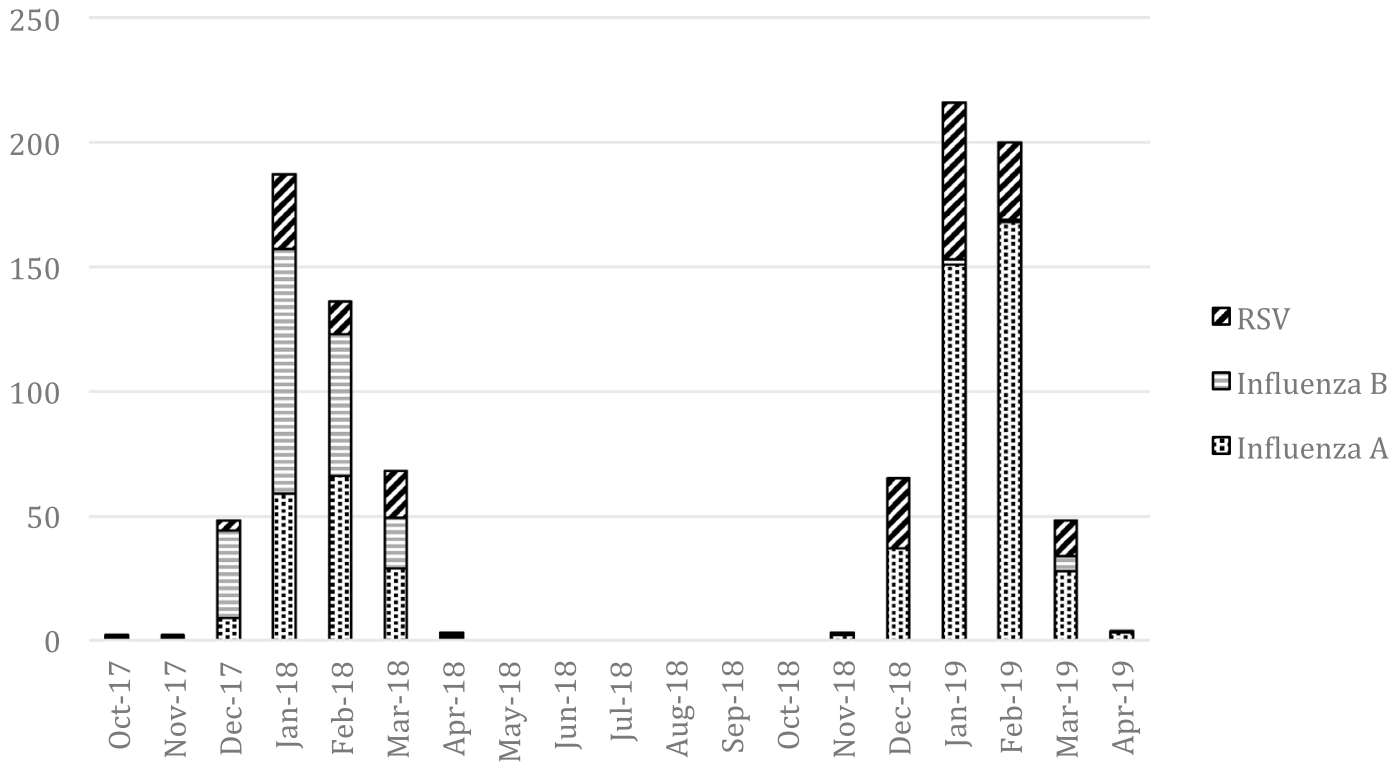

Fig. 2 Monthly cumulative cases of LRTI-related viral isolates $(n=984)$

Table 2 Prevalence of comorbidities and their associations with isolated viruses $(\% / n)$

\begin{tabular}{llllllc}
\hline & Influenza A & H1N1 & H3N2 & Influenza B & RSV & $P$ \\
\hline Male & $61.3 \%(291)$ & $61.5 \%(93)$ & $48.8 \%(144)$ & $47.7 \%(105)$ & $38.1 \%(79)$ & $<\mathbf{0 . 0 0 1}$ \\
Age (median; [IQR]) & $74[61-83]$ & $66[64-89]$ & $78[68-85]$ & $75[65-83]$ & $78[64-86]$ & $<\mathbf{0 . 0 0 1}$ \\
Smoking & $16.9 \%(94)$ & $19.9 \%(30)$ & $14.2 \%(42)$ & $13.6 \%(30)$ & $13.6 \%(30)$ & 0.35 \\
Diabetes & $24.6 \%(58)$ & $16.6 \%(25)$ & $28.1 \%(83)$ & $26.4 \%(58)$ & $26.5 \%(55)$ & 0.05 \\
CKD & $14.9 \%(83)$ & $11.2 \%(17)$ & $15.6 \%(46)$ & $13.6 \%(30)$ & $21.2 \%(44)$ & 0.05 \\
HF & $27.4 \%(153)$ & $15.2 \%(23)$ & $34.6 \%(102)$ & $29.6 \%(65)$ & $38.7 \%(80)$ & $<\mathbf{0 . 0 0 1}$ \\
CLD & $3.8 \%(21)$ & $2.0 \%(3)$ & $4.8 \%(14)$ & $3.6 \%(8)$ & $2.4 \%(5)$ & 0.37 \\
COPD/asthma & $26.7 \%(149)$ & $24.5 \%(37)$ & $30.2 \%(89)$ & $26.8 \%(59)$ & $35.5 \%(78)$ & $\mathbf{0 . 0 2}$ \\
ILD & $0.9 \%(5)$ & $0.7 \%(1)$ & $0.7 \%(2)$ & $1.8 \%(4)$ & $2.9 \%(6)$ & 0.17 \\
OSA/OHS & $4.3 \%(24)$ & $9.3 \%(14)$ & $3.1 \%(9)$ & $3.6 \%(8)$ & $8.2 \%(17)$ & $\mathbf{0 . 0 0 8}$ \\
Solid transplant & $2.2 \%(12)$ & $4.0 \%(6)$ & $1.7 \%(5)$ & $1.4 \%(3)$ & $3.2 \%(7)$ & 0.25 \\
Solid neoplasm & $5.8 \%(32)$ & $5.3 \%(8)$ & $6.4 \%(19)$ & $5.0 \%(11)$ & $7.7 \%(16)$ & 0.66 \\
Hematological neoplasm \\
HIV & $11.0 \%(61)$ & $15.2 \%(23)$ & $8.1 \%(24)$ & $12.3 \%(27)$ & $12.6 \%(26)$ & 0.13 \\
& $2.0 \%(11)$ & $2.0 \%(3)$ & $2.7 \%(8)$ & $3.6 \%(8)$ & $1.0 \%(2)$ & 0.32 \\
\hline
\end{tabular}

Bold values indicate statistically significant $P$ values $(P<0.05)$

Statistical tests (Chi-2 and Kruskal-Wallis) excluded patients infected with non-subtyped Influenza A $(n=875)$ isolated microorganisms and proportions of clinical characteristics in patients harbouring them $(\% / n)$
Univariate associations between clinical characteristics, microorganisms and outcomes are presented in Table 3. As depicted in Fig. 3a, b, on multivariate analysis, H1N1 was predictive of both higher risk of death (aOR 2.0 [1.2-3.4] $p=0.008)$ and IMV (aOR $5.1[3.0-8.5] p<0.001) .1$

There was no crude statistically significant difference in length of stay between Influenza A, Influenza B and
RSV, but this outcome was significantly higher for patients infected with H1N1 when compared to H3N2 (Fig. 4a, b). 
Table 3 Univarate associations between clinical characteristics, microorganisms and outcomes

\begin{tabular}{llllcll}
\hline & NIV & & IMV & $P$ & Death & $P$ \\
\hline Male & $1.1[0.8-1.6]$ & 0.5 & $1.9[1.2-3.0]$ & $\mathbf{0 . 0 0 9}$ & $1.2[0.8-1.8]$ & 0.4 \\
Age & $1.0[1.0-1.0]$ & 0.07 & $0.96[0.95-0.97]$ & $<\mathbf{0 . 0 0 1}$ & $1.01[1.0-1.03]$ & 0.05 \\
Diabetes & $1.4[1.0-2.1]$ & 0.09 & $1.5[0.9-2.4]$ & 0.1 & $1.2[0.74-1.9]$ & 0.5 \\
CKD & $1.3[0.8-2.1]$ & 0.2 & $1.2[0.7-2.2]$ & 0.6 & $1.4[0.8-2.3]$ & 0.2 \\
HF & $1.2[0.8-1.8]$ & 0.3 & $0.6[0.3-1.0]$ & 0.05 & $1.0[0.6-1.5]$ & 1.0 \\
CLD & $0.8[0.3-2.4]$ & 0.8 & $0.7[0.2-2.9]$ & 0.6 & $0.3[0.0-1.9]$ & 0.2 \\
COPD/Asthma & $2.3[1.6-3.4]$ & $<\mathbf{0 . 0 0 1}$ & $1.1[0.7-1.9]$ & 0.6 & $1.1[0.7-1.7]$ & 0.8 \\
ILD & $2.3[0.7-7.5]$ & 0.2 & $2.8[0.8-10.1]$ & 0.1 & $1.3[0.3-5.9]$ & 0.7 \\
OSA/OHS & $7.2[4.0-13.0]$ & $<\mathbf{0 . 0 0 1}$ & $2.3[1.0-5.0]$ & $\mathbf{0 . 0 4}$ & $2.0[1.0-4.3]$ & 0.07 \\
Solid organ transplant & $1.4[0.5-4.3]$ & 0.5 & {$[0.8-7.6]$} & 0.1 & $0.9[0.2-2.7]$ & 0.8 \\
Solid neoplasm & $0.9[0.4-1.9]$ & 0.7 & $0.6[0.2-1.9]$ & 0.4 & $\mathbf{2 . 3}[\mathbf{1 . 2}-\mathbf{4 . 6}]$ & $\mathbf{0 . 0 1}$ \\
Hematological neoplasm & $0.6[0.3-1.2]$ & 0.2 & $1.0[0.5-2.1]$ & 0.9 & $1.4[0.8-2.4]$ & 0.3 \\
HIV & $1.6[0.5-4.6]$ & 0.5 & - & - & $0.4[0.1-3.2]$ & 0.4 \\
Influneza A & $1.0[0.7-1.4]$ & 0.9 & $1.4[0.9-2.3]$ & 0.1 & $0.9[0.6-1.4]$ & 0.8 \\
H1N1 & $2.6[1.7-4.0]$ & $<\mathbf{0 . 0 0 1}$ & $6.0[3.7-9.7]$ & $<\mathbf{0 . 0 0 1}$ & $1.8[1.1-3.0]$ & $\mathbf{0 . 0 2}$ \\
H3N2 & $0.4[0.3-0.7]$ & $\mathbf{0 . 0 0 1}$ & $0.2[0.1-0.5]$ & $<\mathbf{0 . 0 0 1}$ & $0.6[0.4-1.1]$ & 0.08 \\
Influenza B & $0.7[0.4-1.1]$ & 0.1 & $09[0.5-1.6]$ & 0.7 & $0.9[0.6-1.5]$ & 0.8 \\
RSV & $1.5[1.0-2.2]$ & 0.08 & $0.6[0.3-1.2]$ & 0.1 & $1.2[0.7-1.9]$ & 0.5 \\
\hline
\end{tabular}

Bold values indicate statistically significant $P$ values $(P<0.05)$
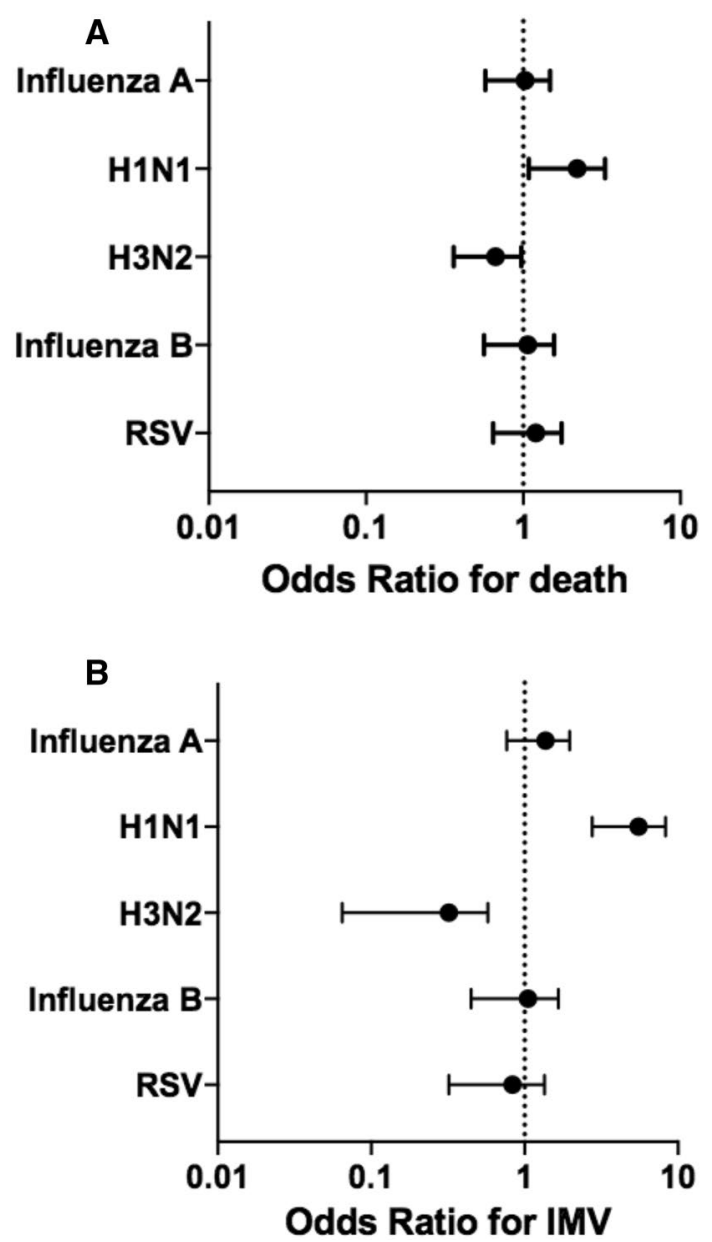

Fig. 3 Adjusted odds ratio for death (a) and invasive mechanical ventilation (IMV) (b) according to microorganism

\section{Discussion}

In this study, Influenza A H1N1 demonstrated higher virulence as it was an independent risk factor for both death and IMV among patients admitted with seasonal viral LRTI's (Fig. 3). It was also associated with higher rates of NIV, and lastly, with a significantly longer length of stay when using H3N2 as a comparator (Fig. 4b).

Influenza infection trends observed in this study (Fig. 2) generally follow the pattern documented in surveillance data from the countries represented, both in terms of incidence peak and Influenza types isolated. Whereas during the 2017-2018 season, the prevailing type was Influenza B, during the 2018-2019, it was Influenza A, predominantly subtype H1N1 in Cyprus [12], roughly evenly H1N1 and H3N2 in Italy [13] and predominantly H3N2 in Portugal [14].

Patients presenting with Influenza A H1N1 were younger (albeit mostly elderly), less likely to be female or have comorbidities such as diabetes or heart failure. The association of lower age and Influenza A H1N1 infection has been observed in large datasets from 2010 to 2019 in Greece [15] and from 2009 to 2015 in an international cohort [16]. The comorbidities mentioned could have been a confounder for lower age in our study. Early post-pandemic sero-prevalence data from 2009 showed that a proportion of older adults had preexisting cross-reactive antibodies to Influenza A H1N1 2009 pandemic strain, possibly owing to immunity to infection as a result of exposure to similar viruses in early life 

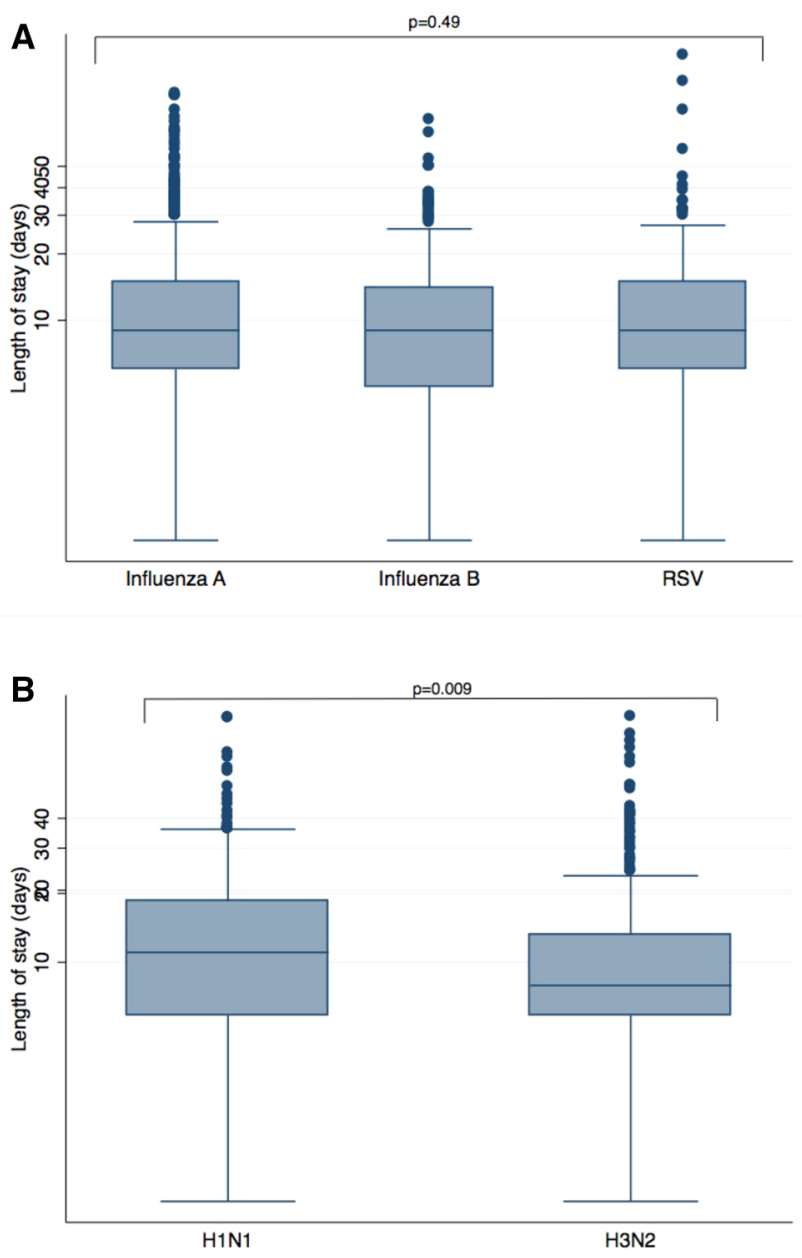

Fig. 4 Length of stay according to microorganism. a Influenza A, Influenza B and Respiratory Sincytial Virus (RSV). b Influenza A subtypes $\mathrm{H} 1 \mathrm{~N} 1$ and $\mathrm{H} 3 \mathrm{~N} 2$

[17]. This is a possible explanation for the association of lower age and infection with this viral subtype, which since 2009 stems from that year's pandemic strain in replacement for the previous lineages [18].

On the other hand, RSV was associated with higher age and comorbidities such as CKD, heart failure and COPD or asthma. This finding is in line with research published over the last decade indicating higher incidence of RSVrelated disease requiring medical attention and/or hospital admission among elderly patients [19-21], even though its association with heart failure and COPD has been found in some studies [19, 20] but not it others [21].

Regarding the association of comorbidities with outcomes, both older age and solid malignancy were risk factors for mortality, while male and younger patients were more likely to be submitted to IMV (Table 3). This apparent mismatch between age and these two outcomes could be explained by older patients presenting with increased frailty and advanced chronic organ failure rendering IMV unsuitable for a proportionate approach to their needs of care. Patients with chronic obstructive pulmonary disease (COPD) or asthma and obesity-hypoventilation syndrome (OHS) or obstructive sleep-apnea (OSA) were more prone to be submitted to NIV. Two possible explanations underlie this finding. First, these broad subsets of patients often present with acute hypercarbic respiratory failure in the context of an acute exacerbation for which a viral agent such as Influenza or RSV might be a trigger [20, 22]. NIV has been demonstrated to be a successful intervention in dealing with such respiratory failure phenotypes, avoiding the hardships and complications of IMV [23]. Second, some of these patients are already on chronic ambulatory bi-level positive airway pressure (BiPAP) ventilation therapy and its use during admission may merely represent the continuation of usual care. The fact that we were not able to ascertain which patients these circumstances could have applied to, so as to exclude them from this subanalysis, represents a limitation.

The main findings of our study suggest an overall higher virulence of Influenza A H1N1 independently of patients' age or preexisting comorbidities. Most research in hospital settings and using national surveillance data conducted after the appearance of the 2009 pandemic H1N1 strain suggests higher severity of this subtype. Similar observations hold both in terms of mortality [15, 24-26] and in terms of inhospital severity outcomes such as ICU admission $[15,16$, 24, 26, 27]. Minchole et al. [24] also found a higher association of Influenza A H1N1 with pneumonia as we did, implying the higher infection of lower respiratory epithelium cells as one possible pathophisiological mechanism underlying higher clinical severity [28]. The only conflicting evidence available so far stems from a multi-hospital cohort study conducted during the 2015-2016 season in Istanbul, showing a higher case fatality rate for Influenza A H3N2 [29].

The fact that the agent found to be most virulent (Influenza A H1N1) was over represented in lower aged healthier patients suggests these may be at higher risk for a more complicated course of disease when admitted for a viral LRTI, which can be at odds with many clinicians' perceptions. This may have implications on Influenza vaccination research and coverage recommendations. Finally, the burden of morbidity and mortality of RSV observed in this study and other pieces of research encourages vaccine development efforts [30].

Limitations of this study include missing subtyping data on some Influenza A isolates. Furthermore, data on Influenza B lineages or neuraminidase inhibitor resistance was not available from patient isolates, although the former can be inferred from national surveillance programs [12-14]. As the study focused only on hospital admissions, it cannot provide data on non-admitted mild or asymptomatic forms of disease and hence a true estimation of case-fatality rates is not possible. Finally, we did not systematically collect data on frailty, which could impact outcomes and IMV decisions. 
Also, vaccination status and bacterial co-infection were not assessed. Nevertheless, the sample included in our study is a real-life cohort representative of the patient population admitted for LRTI's in southern Europe and middle-to-highincome settings with temperate climates. The use of IMV as an outcome instead of ICU admission reduces potential biases created by different local care guidelines and ICU capacities, in exchange for a hard outcome, which is a strong surrogate for severe respiratory failure. Even though data on other respiratory viruses were not sought for, the three microorganisms studied represent the agents most broadly available to clinicians in standard care conditions before the onset of the COVID-19 pandemic.

In conclusion, Influenza A H1N1 may be an independent predictor of higher mortality and IMV rates among patients hospitalized for viral LRTI's, even though its infection disproportionately affects younger and healthier patients. This knowledge combined with surveillance data on the onset of winter seasons may help to forecast the burden of severe LRTI disease and guide planning the allocation of healthcare resources. Further research is needed on RSV vaccine development and on the need to broaden Influenza vaccination coverage to groups usually deemed not to be at risk.

Acknowledgements The authors would like to thank Alberto Clementsen Vaz for the help in graphic conception and design and Dr. Despo Pieridou for enabling access to data.

Funding This research did not receive any specific grant from funding agencies in the public, commercial, or not-for-profit sectors.

\section{Compliance with ethical standards}

Ethics approval The study was approved by the institutional review board of the coordinating centre (Centro Hospitalar de Lisboa Central, number 762_2019) and by the Cyprus National Bioethics Commitee (EEBK EP 2019.01.137).

Consent to participate Obtaining of consent was waived by the institutional review board as the study used de-identified aggregated data.

Conflict of interests The authors declare they have no conflict of interests.

\section{References}

1. Lozano R, Naghavi M, Foreman K, et al. Global and regional mortality from 235 causes of death for 20 age groups in 1990 and 2010: a systematic analysis for the Global Burden of Disease Study 2010. Lancet. 2012;380:2095-128. https://doi.org/10.1016/ S0140-6736(12)61728-0.

2. Iuliano AD, Roguski KM, Chang HH, et al. Estimates of global seasonal influenza-associated respiratory mortality: a modelling study. Lancet. 2018;391:1285-300. https://doi.org/10.1016/S0140 -6736(17)33293-2.
3. Sooryanarain H, Elankumaran S. Environmental role in influenza virus outbreaks. Annu Rev AnimBiosci. 2015;3:347-73. https:// doi.org/10.1146/annurev-animal-022114-111017.

4. Thompson WW, Shay DK, Weintraub E, et al. Influenza-associated hospitalizations in the United States. JAMA. 2004;292:133340. https://doi.org/10.1001/jama.292.11.1333.

5. Park JE, Ryu Y. Transmissibility and severity of influenza virus by subtype. Infect Genet Evol. 2018;65:288-92. https://doi. org/10.1016/j.meegid.2018.08.007.

6. Biggerstaff M, Cauchemez S, Reed C, Gambhir M, Finelli L. Estimates of the reproduction number for seasonal, pandemic, and zoonotic influenza: a systematic review of the literature. BMC Infect Dis. 2014;14:480. https://doi. org/10.1186/1471-2334-14-480.

7. Branche AR, Falsey AR. Respiratory syncytial virus infection in older adults: an under-recognized problem. Drugs Aging. 2015;32:261-9. https://doi.org/10.1007/s40266-015-0258-9.

8. Volling C, Hassan K, Mazzulli T, et al. Respiratory syncytial virus infection-associated hospitalization in adults: a retrospective cohort study. BMC Infect Dis. 2014;14:665. https://doi. org/10.1186/s12879-014-0665-2.

9. Topoulos S, Giesa C, Gatermann S, Fussen R, Lemmen S, Ewig $S$. Analysis of acute respiratory infections due to influenza virus A, B and RSV during an influenza epidemic 2018. Infection. 2019;47:425-33. https://doi.org/10.1007/s15010-018-1262-x.

10. Huang C, Wang Y, Li X, et al. Clinical features of patients infected with 2019 novel coronavirus in Wuhan, China [published correction appears in Lancet. 2020 Jan 30]. Lancet. 2020;395:497-506. https://doi.org/10.1016/S0140 -6736(20)30183-5.

11. Wu X, Cai Y, Huang X, et al. Co-infection with SARS-CoV-2 and Influenza A virus in patient with pneumonia, China. Emerg Infect Dis. 2020;26:1324-6. https://doi.org/10.3201/eid2606.200299.

12. Unit for Surveillance and Control of Communicable diseases, Medical and Public Health Services, Ministry of Health, Nicosia, Cyprus. Seasonal Influenza Cyprus 2017-2019 (unpublished data).

13. Bella A, Castrucci MR. Integrated Influenza surveillance in Italy. 2019. https://www.epicentro.iss.it/ben/2019/luglio-agosto/sorve glianza-integrata-influenza-2018-19.

14. Instituto Nacional de Saúde Doutor Ricardo Jorge. Portuguese national flu surveillance program. 2019. www.insa.min-saude.pt/ wp-content/uploads/2019/10/RelatorioPNVG_2018-2019.pdf.

15 Lytras T, Andreopoulou A, Gkolfinopoulou K, Mouratidou E, Tsiodras S. Association between type-specific influenza circulation and incidence of severe laboratory-confirmed cases; which subtype is the most virulent? Clin Microbiol Infect. 2019. https:// doi.org/10.1016/j.cmi.2019.11.018.

16. Dwyer DE, Lynfield R, Losso MH, et al. Comparison of the outcomes of individuals with medically attended influenza A and $\mathrm{B}$ virus infections enrolled in 2 international cohort studies over a 6-year period: 2009-2015. Open Forum Infect Dis. 2017;4:ofx212. https://doi.org/10.1093/ofid/ofx212.

17. Hancock K, Veguilla V, Lu X, et al. Cross-reactive antibody responses to the 2009 pandemic H1N1 influenza virus. N Engl J Med. 2009;361:1945-52. https://doi.org/10.1056/NEJMoa0906 453.

18 Krammer F, Smith GJD, Fouchier RAM, et al. Influenza. Nat Rev Dis Primers. 2018;4:3. https://doi.org/10.1038/s4157 2-018-0002-y.

19. Kwon YS, Park SH, Kim MA, et al. Risk of mortality associated with respiratory syncytial virus and influenza infection in adults. BMC Infect Dis. 2017;17:785. https://doi.org/10.1186/s1287 9-017-2897-4.

20. Ackerson B, Tseng HF, Sy LS, et al. Severe morbidity and mortality associated with respiratory syncytial virus versus 
influenza infection in hospitalized older adults. Clin Infect Dis. 2019;69:197-203. https://doi.org/10.1093/cid/ciy991.

21. Sundaram ME, Meece JK, Sifakis F, Gasser RA Jr, Belongia EA. Medically attended respiratory syncytial virus infections in adults aged $\geq 50$ years: clinical characteristics and outcomes. Clin Infect Dis. 2014;58:342-9. https://doi.org/10.1093/cid/cit767.

22. Zwaans WA, Mallia P, van Winden ME, Rohde GG. The relevance of respiratory viral infections in the exacerbations of chronic obstructive pulmonary disease - a systematic review. J ClinVirol. 2014;61:181-8. https://doi.org/10.1016/j.jcv.2014.06.025.

23. Osadnik CR, Tee VS, Carson-Chahhoud KV, Picot J, Wedzicha JA, Smith BJ. Non-invasive ventilation for the management of acute hypercapnic respiratory failure due to exacerbation of chronic obstructive pulmonary disease. Cochrane Database Syst Rev. 2017;7:CD004104. https://doi.org/10.1002/14651858.CD004 104.pub4.

24. Minchole E, Figueredo AL, Omeñaca M, et al. Seasonal influenza A H1N1pdm09 virus and severe outcomes: a reason for broader vaccination in non-elderly, at-risk people. PLoS ONE. 2016;11:e0165711. https://doi.org/10.1371/journal.pone.0165711.

25. Chaves SS, Aragon D, Bennett N, et al. Patients hospitalized with laboratory-confirmed influenza during the 2010-2011 influenza season: exploring disease severity by virus type and subtype. J Infect Dis. 2013;208:1305-14. https://doi.org/10.1093/infdis/jit31 6.
26. Caini S, Kroneman M, Wiegers T, El Guerche-Séblain C, Paget J. Clinical characteristics and severity of influenza infections by virus type, subtype, and lineage: A systematic literature review. Influenza Other Respir Viruses. 2018;12:780-92. https://doi. org/10.1111/irv.12575.

27. Boddington NL, Verlander NQ, Pebody RG. Developing a system to estimate the severity of influenza infection in England: findings from a hospital-based surveillance system between 2010/2011 and 2014/2015. Epidemiol Infect. 2017;145:1461-70. https://doi. org/10.1017/S095026881700005X.

28. Guarner J, Falcón-Escobedo R. Comparison of the pathology caused by $\mathrm{H} 1 \mathrm{~N} 1, \mathrm{H} 5 \mathrm{~N} 1$, and $\mathrm{H} 3 \mathrm{~N} 2$ influenza viruses. Arch Med Res. 2009;40:655-61. https://doi.org/10.1016/j.arcme d.2009.10.001.

29. Tekin S, Keske S, Alan S, et al. Predictors of fatality in influenza A virus subtype infections among inpatients in the 2015-2016 season. Int J Infect Dis. 2019;81:6-9. https://doi.org/10.1016/j. ijid.2019.01.005.

30. Cicconi P, Jones C, Sarkar E, et al. First-in-human randomized study to assess the safety and immunogenicity of an investigational respiratory syncytial virus (RSV) vaccine based on chimpanzee-adenovirus-155 viral vector-expressing RSV fusion, nucleocapsid, and antitermination viral proteins in healthy adults. Clin Infect Dis. 2020;70:2073-81. https://doi.org/10.1093/cid/ ciz653. 